\title{
Bundling Mobile Base Station and Wireless Energy Transfer: Modeling and Optimization
}

\author{
Liguang Xie \\ Yi Shi \\ Y. Thomas Hou* \\ Wenjing Lou \\ Hanif D. Sherali \\ Scott F. Midkiff \\ Virginia Polytechnic Institute and State University, USA
}

\begin{abstract}
Wireless energy transfer is a promising technology to fundamentally address energy and lifetime problems in a wireless sensor network (WSN). On the other hand, it has been well recognized that a mobile base station has significant advantages over a static one. In this paper, we study the interesting problem of co-locating the mobile base station on the wireless charging vehicle (WCV). The goal is to minimize energy consumption of the entire system while ensuring none of the sensor nodes runs out of energy. We develop a mathematical model for this complex problem. Instead of studying the general problem formulation (OPT-t), which is time-dependent, we show that it is sufficient to study a special subproblem (OPT-s) which only involves space-dependent variables. Subsequently, we develop a provably near-optimal solution to OPT-s. The novelty of this research mainly resides in the development of several solution techniques to tackle a complex problem that is seemingly intractable at first glance. In addition to addressing a challenging and interesting problem in a WSN, we expect the techniques developed in this research can be applied to address other related networking problems involving time-dependent movement, flow routing, and energy consumption.
\end{abstract}

\section{INTRODUCTION}

Recently, wireless energy transfer based on magnetic resonant coupling was shown to be a promising technology to fundamentally address energy and lifetime problems in a wireless sensor network (WSN) [13], [14]. Compared to other wireless energy transfer technologies such as electromagnetic radiation [3], [11], magnetic resonant coupling [5], [6] enjoys significant advantages. These advantages include higher wireless power transfer efficiency, immunity to the neighboring environment, and no requirement of line-of-sight or any alignment (omnidirectional) [16]. Nevertheless, due to the potential large geographical coverage of a WSN, it is still necessary to employ a vehicle (called wireless charging vehicle (WCV) in [13], [14]) to carry the charging device and bring it to a reasonable distance close to the sensor nodes. Note that in [13], [14], although the WCV is mobile, the base station in the WSN (sink node for all sensing data) is fixed.

On the other hand, it has been well recognized that a mobile base station can achieve significant energy saving and network lifetime extension [8], [10], [17]. Given that mobile base station also needs a vehicle to carry, a natural question to ask becomes: Is it possible to have the base station co-

\footnotetext{
*For correspondence, please contact Tom Hou (thou@vt.edu).
}

locate on the same vehicle used for wireless energy transfer? ${ }^{1}$ This is the main motivation of this investigation.

In this paper, we explore problems centered around bundling the mobile base station and the WCV. When there is no ambiguity, we still call the combined two systems as WCV. We envision the WCV starts from its home service station, travels along a pre-planned path and returns to its home service station at the end of a trip. While traveling on its path, the WCV can make a number of stops and charge sensor nodes near those stops. At any time, all data collected by the sensor nodes are relayed (via multi-hop) to the mobile base station (on WCV). Apparently this is a very complex system, involving variables across multiple dimensions - time, space, and energy. A basic requirement is that by employing wireless energy transfer, none of the sensor nodes run out of energy while all sensing data are relayed to the base station in real time. A more aggressive goal is to minimize energy consumption of the entire system (see Section IV for more details).

This is a challenging optimization problem that involves several subproblems, each of which is interesting on its own. First, the WCV's movement behavior needs to be optimized, which will tell us where the WCV will make stops along its path and how long it will stay at each stop. Second, the flow routing among sensor nodes need to be optimized, which depends on the location of WCV (and time). Finally, the energy transfer behavior of the WCV depends on its stopping locations and their distances to neighboring sensor nodes. Apparently, these subproblems are tightly coupled together (both in time/space and under a global optimization objective).

The main contributions of this paper are as follows:

- We develop a mathematical model for co-locating the mobile base station on the WCV. This includes energy criteria to ensure that the energy level at each sensor node never falls below some minimum threshold, and a general optimization problem formulation (OPT- $t$ ) involving the WCV's stopping behavior, energy charging, and data flow routing. We also show that the goal of minimizing energy consumption associated with the

\footnotetext{
${ }^{1}$ Although two separate vehicles may be employed (one is WCV and the other for mobile base station), the costs (both equipment and energy) associated with the two vehicles will be much higher.
} 
WCV is equivalent to maximizing the fraction of the WCV's vacation time at the service station.

- The general problem OPT-t has a time-dependent formulation, which is difficult to devise any solution. We show that a special case of OPT-t, where data flow routing and energy consumption of sensor nodes only depend on the WCV's location, can in fact offer the same optimal objective value as that for OPT-t. Therefore, we propose to study this simpler formulation, denoted as OPT-s, that only involves location-dependent variables.

- For problem OPT-s, we develop an $(1-\epsilon)$-optimal solution with any desired level of accuracy $\epsilon$. Our solution involves discretizing path into a finite number of segments and representing each segment as a logical point. By exploiting the worst case and the best case representation for each logical point, we can find a lower bound and an upper bound for OPT-s by solving two linear programs (LPs). Depending on the required accuracy, we show how to discretize segments into smaller segments. We prove that the gap between the lower and upper bounds will close as the number of iterations increases and we will eventually obtain an $(1-\epsilon)$-optimal solution.

It is worth contrasting this paper with [10] in terms of problem scope and solution techniques. In [10], only a mobile base station problem was investigated while in this paper, we investigate the WCV and mobile base station co-location problem. In [10], it was assumed that the time for the base station to move from one point to another is negligible while in this paper we explicitly consider the traveling time in problem formulation and solution. Further, in this paper, we generalize the "fictitious cost point" concept proposed in [10], which only considered energy consumption. That is, the so-called "logical point" concept in this paper considers both energy reception and energy consumption.

The remainder of this paper is organized as follows. In Section II, we describe some basic models for the WCV's stopping behavior, flow routing, and energy charging and consumption in a WSN. Section III presents energy criteria to ensure that each sensor node is always operational. A general optimization formulation (OPT-t) is given in Section IV. In Section V, we show that we can study a special case of OPT-t that only involves space-dependent formulation, which we denote as OPT-s. Section VI presents an $(1-\epsilon)$-optimal solution to OPT-s. Section VII presents numerical results and Section VIII concludes this paper.

\section{Basic Network ANd Energy Models}

In this section, we present some basic models for the WCV's stopping behavior, flow routing, and energy charging and consumption in a WSN.

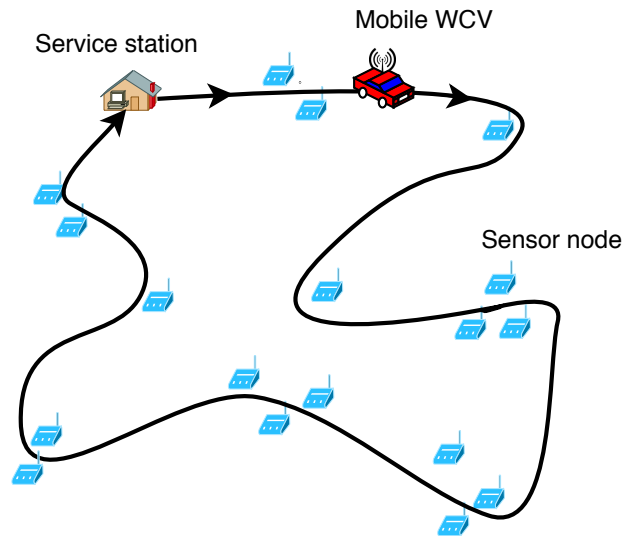

Fig. 1. An example WSN with a mobile WCV.

\section{A. WCV and Travel Path}

Suppose we have a sensor network $\mathcal{N}$ deployed over a two-dimensional area, with the location of each node $i \in \mathcal{N}$ being $\left(x_{i}, y_{i}\right)$. A WCV is employed to charge sensor nodes in the network. This WCV starts from a service station, travels along a pre-planned path in the area and returns to the service station at the end of its trip. While on its path, the WCV can make a number of stops and charge sensor nodes near those stops (see Fig. 1). The detailed energy charging model will be described in Section II-D.

Denote $\mathcal{P}$ as the traveling path and $\tau$ as the total amount of time for the WCV to complete the trip. Then $\tau$ includes three components:

- The total traveling time along path $\mathcal{P}$ is $D_{\mathcal{P}} / V$, where $D_{\mathcal{P}}$ is the distance of path $\mathcal{P}$ and $V$ is the traveling speed of WCV.

- The vacation time $\tau_{\text {vac }}$, which refers to the amount of time that the WCV stays at the service station (at point $p_{\text {vac }}$ ) before leaving for the next trip.

- The total stopping time along path $\mathcal{P}$. Denote $\omega(p)$ as the aggregate amount of time the WCV stops at point $p \in \mathcal{P}$. Since the WCV may stops at $p$ more than once during $\tau$, we have:

$$
\omega(p)=\int_{\{t \in[0, \tau]:(x, y)(t)=p\}} 1 \mathrm{~d} t,
$$

where $(x, y)(t)$ is the location of the WCV at time $t$. Then the total stopping time is $\sum_{p \in \mathcal{P}, p \neq p_{\text {vac }}}^{\omega(p)>0} \omega(p)$.

Then we have:

$$
\tau=\frac{D_{\mathcal{P}}}{V}+\tau_{\text {vac }}+\sum_{p \in \mathcal{P}, p \neq p_{\text {vac }}}^{\omega(p)>0} \omega(p) .
$$

\section{B. Mobile Base Station and Data Flow Routing}

As discussed, we assume that the base station is co-located at the WCV. Therefore, the base station is also mobile and 
serves as the sink node for all data collected by the WSN. To conserve energy, multi-hop data routing is employed among the sensor nodes in the network.

Suppose each sensor node $i$ produces its local data with a constant rate $G_{i}$ (in b/s), $i \in \mathcal{N}$. Denote $f_{i j}(t)$ and $f_{i B}(t)$ as the flow rates from node $i$ to node $j$ and the base station at time $t$, respectively. Then we have the following flow balance at each sensor node $i$ :

$$
\sum_{k \in \mathcal{N}}^{k \neq i} f_{k i}(t)+G_{i}=\sum_{j \in \mathcal{N}}^{j \neq i} f_{i j}(t)+f_{i B}(t) \quad(i \in \mathcal{N}) .
$$

Note that we are dealing with real-time flow routing rather than delay-tolerant data MULEs [9] or message ferry [19] type of communications.

\section{Sensor Energy Consumption}

At a sensor node, we assume that communications are the dominant source for the node's energy consumption. Denote $C_{i j}$ as the energy consumption rate for transmitting one unit of data flow from sensor node $i$ to sensor node $j$. Then $C_{i j}$ can be modeled as [2], [4]:

$$
C_{i j}=\beta_{1}+\beta_{2} D_{i j}^{\alpha},
$$

where $D_{i j}$ is the distance between nodes $i$ and $j, \beta_{1}$ and $\beta_{2}$ are constant terms, and $\alpha$ is the path loss index. Suppose all sensor nodes are stationary. Then the inter-sensor distance $D_{i j}$ and $C_{i j}$ are all constants.

Denote $C_{i B}(p(t))$ as the energy consumption rate for transmitting one unit of data flow from sensor node $i$ to base station $B$ when $B$ is at location $p(t)$. Then we have

$$
C_{i B}(p(t))=\beta_{1}+\beta_{2}\left[\sqrt{\left(x(t)-x_{i}\right)^{2}+\left(y(t)-y_{i}\right)^{2}}\right]^{\alpha},
$$

where $(x(t), y(t))$ and $\left(x_{i}, y_{i}\right)$ are the coordinates of $p(t)$ and node $i$, respectively. Note that unlike $C_{i j}$ 's, which are all constants, $C_{i B}(p(t))$ varies with the base station's location over time.

Denote $\rho$ as the rate of energy consumption for receiving one unit of data flow. Then the total energy consumption rate for both transmission and reception at node $i$, denoted as $r_{i}(t)$, is

$$
r_{i}(t)=\rho \sum_{k \in \mathcal{N}}^{k \neq i} f_{k i}(t)+\sum_{j \in \mathcal{N}}^{j \neq i} C_{i j} \cdot f_{i j}(t)+C_{i B}(p(t)) \cdot f_{i B}(t)
$$

\section{WCV Charging Model}

We assume that the WCV can only perform its charging function when it makes a full stop somewhere along path $\mathcal{P}$ (except $p_{\text {vac }}$ ). Denote $U_{i B}(p)$ as the power reception rate at node $i$ when the WCV is located at $p \in \mathcal{P}$. Denote the efficiency of wireless charging by $\mu\left(D_{i B}(p)\right)$, which is a decreasing function of distance $D_{i B}(p)$. Then the wireless charging model [14] is:

$$
U_{i B}(p)=\left\{\begin{array}{ll}
\mu\left(D_{i B}(p)\right) \cdot U_{\max } & \text { if } D_{i B}(p) \leq D_{\delta} \\
0 & \text { if } D_{i B}(p)>D_{\delta}
\end{array},\right.
$$

where $U_{\max }$ is the maximum output power for a single sensor node and $D_{\delta}$ is the charging range of the WCV, below which wireless charging cannot be performed. In other words, $D_{\delta}$ is defined in a way such that the power reception rate at a sensor node is at least over a threshold value $\delta$. We assume that a sensor node can be charged only if it is within a distance of $D_{\delta}$ from path $\mathcal{P}$.

When multiple nodes receive power from the WCV simultaneously, interference may occur due to resonant coupling between receiving nodes. The interference can be handled by adjusting resonant frequencies at the source and receiving nodes [1].

\section{ENERGY CRITERIA FOR CYCLES}

We assume that the WCV follows a fixed travel schedule along $\mathcal{P}$ with a period of $\tau$. Then we have $p(t)=p(t+k \tau)$ for $0 \leq t \leq \tau, k=1,2, \cdots$. Further, we assume that the flow routing in the network also follows a periodic cycle, i.e., $f_{i j}(t)=f_{i j}(t+k \tau)$ and $f_{i B}(t)=f_{i B}(t+k \tau)$ for $0 \leq t \leq \tau, k=1,2, \cdots$. Then by (5), the energy consumption rate at each node also follows a periodic cycle, i.e., $r_{i}(t)=r_{i}(t+k \tau)$ for $0 \leq t \leq \tau, i \in \mathcal{N}$. Since the WCV's charging behavior only depends on its travel schedule, then the charging behavior also forms a periodic cycle. In summary, the travel schedule of the WCV, its charging behavior along its path, flow routing among the nodes, and energy consumption at each node are all cyclic and repeat themselves with a period of $\tau$.

Suppose that each sensor node has a battery capacity of $E_{\max }$ and is fully charged initially. Denote $E_{\min }$ as the minimum energy at a sensor node battery for it to be operational. We are interested in developing a particular travel cycle so that the energy level at each sensor node at time $t$, denoted as $e_{i}(t), i \in \mathcal{N}$, never falls below $E_{\min }$. In the following, we will offer two constraints for the first cycle. We will show that once these two constraints hold for the first cycle, then $e_{i}(t) \geq E_{\min }$ for $t \geq \tau$, i.e., all future cycles.

The first constraint ensures that $e_{i}(t)$, which starts from $E_{\max }$ at $t=0$, will not fall below $E_{\min }$ at $t=\tau$,

$$
E_{\max }-\left\{\int_{\{t \in[0, \tau]: \omega(p(t))=0\}} r_{i}(t) \mathrm{d} t+\right.
$$

$$
\left.\int_{\left\{t \in[0, \tau]: \omega(p(t))>0, D_{i B}(p(t))>D_{\delta}\right\}} r_{i}(t) \mathrm{d} t\right\} \geq E_{\text {min }} \quad(i \in \mathcal{N}),
$$

where $\int_{\{t \in[0, \tau]: \omega(p(t))=0\}} r_{i}(t) \mathrm{d} t$ is the amount of energy consumed at node $i$ when the WCV is moving along path $\mathcal{P}$ 
while $\int_{\left\{t \in[0, \tau]: \omega(p(t))>0, D_{i B}(p(t))>D_{\delta}\right\}} r_{i}(t) \mathrm{d} t$ is the amount of energy consumed at node $i$ when the WCV is making stops but node $i$ is outside the WCV's charging range.

The second constraint ensures that $e_{i}(t)$, which starts from $E_{\max }$ at $t=0$, will be charged back to $E_{\max }$ before the end of the first cycle $\tau$. We have

$\int_{0}^{\tau} r_{i}(t) \mathrm{d} t \leq \sum_{p \in \mathcal{P}}^{\omega(p)>0, D_{i B}(p) \leq D_{\delta}} U_{i B}(p) \cdot \omega(p) \quad(i \in \mathcal{N})$,

where the left hand side is the amount of energy consumed at node $i$ during $\tau$ and the right hand side is the maximum possible amount of energy received by node $i$ in a cycle. Note that the actual amount of energy received by node $i$ in the first cycle may be less than the right hand side due to potential battery overflow. ${ }^{2}$

Note that (7) and (8) characterize the energy consumption and reception in the first cycle. The following lemma says that if both (7) and (8) hold for the first cycle, then we have $e(t) \geq E_{\min }$ for all cycles.

Lemma 1 (Energy Criteria): If both (7) and (8) are satisfied for the first cycle, then $e_{i}(t) \geq E_{\text {min }}$ for all $t \geq 0$, $i \in \mathcal{N}$.

Lemma 1 can be proved by showing that $e_{i}(t) \geq E_{\min }$ for the second cycle and a node's energy behavior repeats from cycle to cycle (starting at the second cycle). A formal proof of Lemma 1 is given in [15].

\section{PROBLEM Formulation}

In Section III, we explained energy criteria for cycles. Under Lemma 1, we showed that under certain conditions, $e_{i}(t) \geq E_{\text {min }}$ for all $t \geq 0, i \in \mathcal{N}$. In addition to these constraints, we can also consider optimizing some global performance objective. In particular, we want to minimize the rate of energy consumption for the entire system, which encompasses all energy consumption at the WCV as follows: ${ }^{3}$

- The energy consumption rate for carrying the WCV to move along $\mathcal{P}$ during $\tau$ is

$$
\frac{R_{v} \cdot\left[\frac{D_{\mathcal{P}}}{V}+\sum_{p \in \mathcal{P}, p \neq p_{\text {vac }}}^{\omega(p)>0} \omega(p)\right]}{\tau},
$$

where $D_{\mathcal{P}} / V+\sum_{p \in \mathcal{P}, p \neq p_{\text {vac }}}^{\omega(p)>0} \omega(p)$ is the time that the $\mathrm{WCV}$ is at work during $\tau$ (i.e., outside its service station), and $R_{v}$ denotes the energy consumption rate for carrying the WCV in this period. Note that the energy

\footnotetext{
${ }^{2}$ Once a battery is charged to $E_{\max }$, its energy level cannot be further increased.

${ }^{3}$ Note that except their initial energy, the energy consumed in the WSN comes from the WCV.
}

consumed at the WCV when it makes stops along $\mathcal{P}$ should be included as the vehicle's engine is still on.

- The rate of energy transferred from the WCV to the sensor nodes in the network is at most $\frac{\sum_{i \in \mathcal{N}} r_{i}}{\delta / U_{\max }}$, where $\sum_{i \in \mathcal{N}} r_{i}$ is the sum of energy consumption rates at the sensor nodes, and $\delta / U_{\max }$ is the minimum efficiency for effective wireless charging. ${ }^{4}$

To date, the most fuel-efficient vehicle has an energy consumption of $17.4 \mathrm{~kW}-\mathrm{h}$ per $100 \mathrm{~km}$ [12], which indicates that $R_{v}$ is on the order of $1 \mathrm{~kW}$. In contrast, the rate of energy transferred to sensor nodes is roughly on the order of 0.1 to $1 \mathrm{~W}$ (see Section VII). Since the energy consumed to carry the WCV is the dominant source of energy consumption, we only need to consider this dominant part, i.e., minimizing (9). Since $R_{v}$ is a constant factor, we can remove it from the objective function.

It is interesting that, by (2), minimizing $\frac{D_{\mathcal{P}} / V+\sum_{p \in \mathcal{P}, p \neq p_{\text {vac }}}^{\omega(p)>0}(p)}{\tau}$ is equivalent to maximizing $\frac{\tau_{\text {vac }}}{\tau}$, which is the percentage of time that the WCV is on vacation at its service station. This is intuition. Therefore, we have the following optimization problem.

\section{OPT-t:}

$\operatorname{maximize} \quad \frac{\tau_{\mathrm{vac}}}{\tau}$

s.t. Time constraints: (1), (2);

Flow routing constraints: (3);

Energy consumption model: (4), (5);

Energy criteria constraints: (7), (8) . $\tau, \tau_{\text {vac }}, \omega(p) \geq 0,(x, y)(t) \in \mathcal{P} \quad(p \in \mathcal{P}, 0 \leq t \leq \tau)$ $f_{i j}(t), f_{i B}(t), C_{i B}(t), r_{i}(t) \geq 0,(i, j \in \mathcal{N}, i \neq j, 0 \leq t \leq \tau)$.

In this formulation, $\mathcal{P}, D_{\mathcal{P}}, V, G_{i}, \beta_{1}, \beta_{2}, \alpha, x_{i}, y_{i}, \rho, C_{i j}$, $E_{\max }$, and $E_{\min }$ are given a priori, and $U_{i B}(p)$ can be computed by (6). The time intervals $\tau, \tau_{\text {vac }}$, and $\omega(p)$, the WCV's location $(x, y)(t)$, the flow rates $f_{i j}(t)$ and $f_{i B}(t)$, the unit cost rate $C_{i B}(t)$, and the power consumption rate $r_{i}(t)$ are optimization variables. Among these variables, there are three sets of variables that constitute the solution space: (i) the WCV's location (i.e., $(x, y)(t)$ ); (ii) the WCV's sojourn time at each location $p \in \mathcal{P}$ and $p \neq p_{\text {vac }}$ (i.e., $\omega(p)$ ) or vacation time at the service station (i.e., $\tau_{\text {vac }}$ ); (iii) the corresponding flow routing (i.e., $f_{i j}(t)$ and $f_{i B}(t)$ ). Problem OPT-t is a continuous-time nonlinear program [18], and is NP-hard in general.

\section{Downsizing Solution Space: A Location-BASED FORMULATION}

Roadmap. OPT-t is a general formulation of our problem. It is difficult as its variables are time-dependent (e.g.,

${ }^{4}$ From experimental results in [6], the ratio of $\delta$ to $U_{\max }$ can be set as $20 \%$ (see Section VII). 


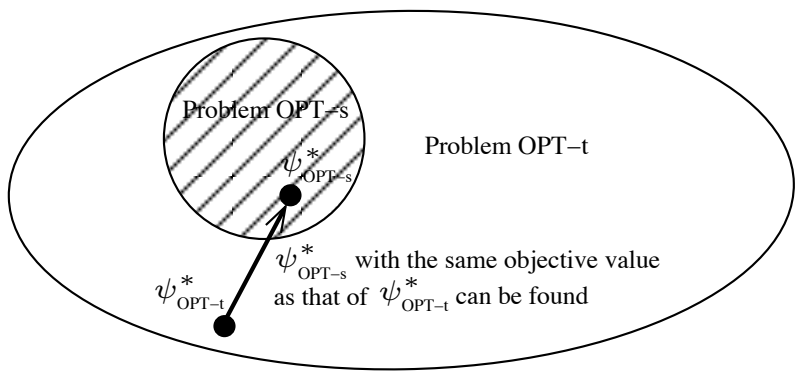

Fig. 2. Solution space for problems OPT-t and OPT-s. $\psi_{\text {OPT-t }}^{*}$ and $\psi_{\text {OPT-s }}^{*}$ are optimal solutions to OPT-t and OPT-s, respectively.

$\left.(x, y)(t), f_{i j}(t)\right)$. In this general formulation, OPT-t allows data flow routing and energy consumption of sensor nodes to vary over time, even when the WCV visits the same location.

In this section, we consider a special case of problem OPT-t, where data flow routing and energy consumption of sensor nodes only depend on WCV's location. That is, as long as the WCV visits a location $p \in \mathcal{P}$, the data flow routing and energy consumption of sensor nodes are the same regardless when the WCV visits this location. This location (space)dependent problem is a special case of Problem OPT-t. We denote this problem as OPT-s. The solution space for OPT-s and OPT-t is shown in Fig. 2, in which the solution space for OPT-s is completely contained in that for OPT-t.

We will show that the optimal objective value for OPT-s is the same as that for OPT-t, despite that its solution space is smaller (Theorem 1). This result is significant as it allows us to study OPT-s, which has a simpler formulation that only involves location-dependent variables.

Location-dependent Formulation. We now formulate OPT-s. First, we need some new notations. Denote $f_{i j}(p)$ and $f_{i B}(p)$ as flow rates from sensor node $i$ to sensor node $j$ and to the base station when the WCV is at location $p \in \mathcal{P}$, respectively. Then (3) is rewritten as

$$
\sum_{k \in \mathcal{N}}^{k \neq i} f_{k i}(p)+G_{i}=\sum_{j \in \mathcal{N}}^{j \neq i} f_{i j}(p)+f_{i B}(p) \quad(i \in \mathcal{N}, p \in \mathcal{P}) .
$$

Similarly, denote $C_{i B}(p)$ and $r_{i}(p)$ as the energy consumption for transmitting one unit of data flow from node $i$ to the base station and the energy consumption rate at node $i$ when the WCV is at location $p \in \mathcal{P}$, respectively. Then (5) can be rewritten as:

$$
\begin{aligned}
r_{i}(p)=\rho \sum_{k \in \mathcal{N}}^{k \neq i} f_{k i}(p)+\sum_{j \in \mathcal{N}}^{j \neq i} C_{i j} \cdot f_{i j}(p) \\
\quad+C_{i B}(p) \cdot f_{i B}(p) \quad(i \in \mathcal{N}, p \in \mathcal{P}) .
\end{aligned}
$$

Now we rewrite (7) and (8) into location-based constraints. We start with (8). In (8), $\int_{0}^{\tau} r_{i}(t) \mathrm{d} t$ can be split into two parts:
- energy consumed when WCV makes stops (including vacation at the service station), which is, $r_{i}\left(p_{\text {vac }}\right) \cdot \tau_{\text {vac }}+$ $\sum_{p \in \mathcal{P}, p \neq p_{\text {vac }}}^{\omega(p)>0} r_{i}(p) \cdot \omega(p)$

- energy consumed when WCV travels along $\mathcal{P}$, i.e.,

$$
\int_{t \in[0, \tau]}^{\omega(p(t))=0} r_{i}(t) \mathrm{d} t=\int_{s \in\left[0, D_{\mathcal{P}}\right]}^{\omega(p(s))=0} r_{i}(p(s)) \frac{d t}{d s} d s,
$$

where $s \in\left[0, D_{\mathcal{P}}\right]$ is the distance traversed by the WCV along $\mathcal{P}$ (starting from its service station), and $p(s)$ is the WCV's location corresponding to $s$. Denote $W=$ $\lim _{\Delta \rightarrow 0} \frac{\Delta t}{\Delta s}$. Then $W=\frac{1}{V}$, and (12) can be rewritten as:

$$
\int_{t \in[0, \tau]}^{\omega(p(t))=0} r_{i}(t) \mathrm{d} t=\int_{s \in\left[0, D_{\mathcal{P}}\right]}^{\omega(p(s))=0} W \cdot r_{i}(p(s)) d s .
$$

Based on the above discussion, (8) can be rewritten as:

$$
\begin{aligned}
r_{i}\left(p_{\text {vac }}\right) \cdot & \tau_{\text {vac }}+\sum_{p \in \mathcal{P}, p \neq p_{\text {vac }}}^{\omega(p)>0} r_{i}(p) \cdot \omega(p) \\
& +\int_{s \in\left[0, D_{\mathcal{P}}\right]}^{\omega(p(s))=0} W \cdot r_{i}(p(s)) d s \\
\leq & \sum_{p \in \mathcal{P}}^{\omega(p)>0, D_{i B}(p) \leq D_{\delta}} U_{i B}(p) \cdot \omega(p) \quad(i \in \mathcal{N}),
\end{aligned}
$$

which is a location-dependent formulation.

Similarly, (7) can be rewritten as:

$$
\begin{aligned}
& r_{i}\left(p_{\text {vac }}\right) \cdot \tau_{\text {vac }}+\sum_{p \in \mathcal{P}, p \neq p_{\text {vac }}}^{\omega(p)>0, D_{i B}(p)>D_{\delta}} r_{i}(p) \cdot \omega(p) \\
& +\int_{s \in\left[0, D_{\mathcal{P}}\right]}^{\omega(p(s))=0} W \cdot r_{i}(p(s)) d s \leq E_{\max }-E_{\min } \quad(i \in \mathcal{N}) .
\end{aligned}
$$

We now have a formulation for OPT-s, which only involves location-dependent variables.

\section{OPT-s:}

$\operatorname{maximize} \frac{\tau_{\text {vac }}}{\tau}$

s.t. Time constraint: (2);

Flow routing constraints: (10);

Energy consumption model: (11);

Energy criteria constraints: (13), (14).

$\tau, \tau_{\text {vac }}, \omega(p) \geq 0 \quad(p \in \mathcal{P})$

$$
f_{i j}(p), f_{i B}(p), r_{i}(p) \geq 0 \quad(i, j \in \mathcal{N}, i \neq j, p \in \mathcal{P}) .
$$

Proof of Equivalence. We now show that the optimal objective value of OPT-s is the same as that for OPT-s (see Fig. 2). For Problem OPT-t, denote $\psi_{\text {OPT-t }}$ and $\psi_{\text {OPT } t}^{*}$ as a feasible solution and an optimal solution, respectively. Similarly, for Problem OPT-s, denote $\psi_{\text {OPT-s }}$ and $\psi_{\text {OPT-s }}^{*}$ as a feasible solution and an optimal solution, respectively.

Theorem 1: The optimal objectives achieved by $\psi_{\text {OPT-S }}^{*}$ and $\psi_{\text {OPT.t }}^{*}$ are identical. 
The proof of this theorem is based on two results. First, we show that the optimal objective achieved by $\psi_{\text {OPT-t }}^{*}$ is no less than that achieved by solution $\psi_{\text {OPT-s }}^{*}$. This is straightforward as OPT-s is a subproblem of OPT-t. Second, we show that the converse is also true. Instead of considering an optimal solution, we will show that for any objective value achieved by a feasible solution $\psi_{\text {OPT-t }}$, we can always find a feasible solution $\psi_{\text {OPT-s }}$, which achieves the same objective value (Lemma 2). If this is true, in the special case when the feasible solution $\psi_{\text {OPT-t }}$ is the optimal solution $\psi_{\text {OPT-t }}^{*}$, the objective by $\psi_{\mathrm{OPT}-\mathrm{t}}^{*}$ can also be achieved by some feasible solution $\psi_{\text {OPT-s }}$.

Lemma 2: Given a feasible $\psi_{\text {OPT-t }}$, we can construct a feasible $\psi_{\text {OPT-s }}$ with the same objective value.

A formal proof of Lemma 2 is given in [15]. Based on Theorem 1, we know that as long as our objective is concerned, it is sufficient to study OPT-s, which has a simpler formulation than OPT-t.

\section{A NeAr-Optimal Solution to Problem OPT-S}

Although OPT-s is simpler than OPT-t, path $\mathcal{P}$ still has infinite number of points. In this section, by discretizing path $\mathcal{P}$ into a finite number of segments and representing each segment as a logical point, we develop a provably $(1-\epsilon)$ near-optimal solution.

\section{A. Basic Idea}

Fig. 3 shows the flow chart of our proposed algorithm. In Step 1, we discretize path $\mathcal{P}$ into $M_{1}$ segments of equal distance, i.e., $D_{\mathcal{P}} / M_{1}$. For each segment, we represent it as a logical point. Once the WCV is within a segment, we say that it is visiting the corresponding logical point, regardless the specific location within this segment. Note that the time that the WCV spends at a logical point includes both its traveling time over this segment as well as stopping time at any point in this segment.

Since each sensor node's energy consumption and charging behaviors depend on the specific location of the WCV within a segment, it is not obvious to characterize these energy behaviors for the corresponding logical point. Fortunately, we find that for the purpose of developing an $(1-\epsilon)$-optimal algorithm, it is sufficient to use the worst case behavior. That is, to characterize a sensor node's energy consumption and charging behaviors when the $\mathrm{WCV}$ is at a logical point, it is sufficient to consider maximum energy consumption and minimum energy charging.

Once we have a worst case representation for each logical point, in Step 3, we can find a lower bound for OPT-s by solving a LP. Also in Step 3, by developing a best case representation for each logical point (following the same

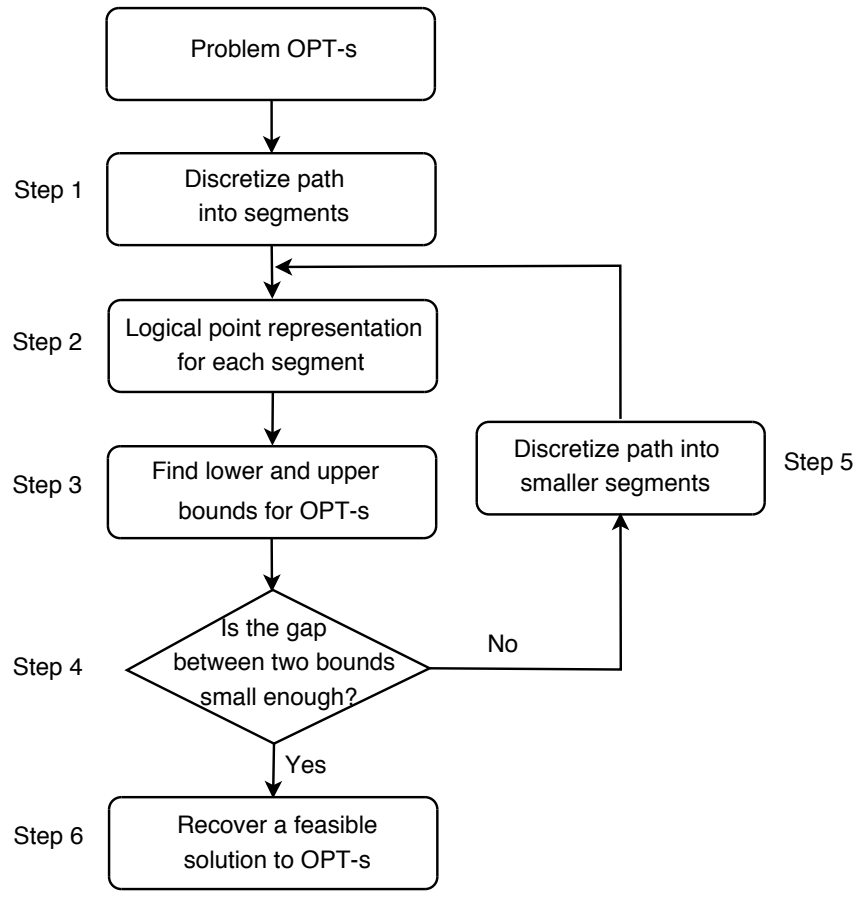

Fig. 3. A flow chart of our algorithm.

token for the worst case representation), we can find an upper bound for OPT-s by solving another LP.

In Step 4, we check the gap between objective values of the lower and upper bounds. If the gap is within $\epsilon$ of the upper bound, we have an $(1-\epsilon)$-optimal solution (corresponding to the lower bound solution, which is feasible); otherwise, we increase the number of segments (Step 5) linearly and return to Step 2.

As the iteration continues, we expect the gap between lower and upper bounds gets closer and closer and we shall have an $(1-\epsilon)$-optimal solution (when the lower bound is within $(1-\epsilon)$ of the upper bound). From the solution corresponding to the final lower bound, we can construct a feasible solution to Problem OPT-s (Step 6).

\section{B. Some Details}

In this section, we give some details on each step of our algorithm.

Path Discretization (Step 1). In the first iteration, path $\mathcal{P}$ is equally divided into $M_{1}$ segments, each of which is indexed in increasing order following the WCV's traveling direction. Denote $\mathcal{S}_{m}$ and $D\left(\mathcal{S}_{m}\right), m=1,2, \ldots, M_{1}$, as the $m$-th segment and its length, with $D\left(\mathcal{S}_{m}\right)=D_{\mathcal{P}} / M_{1}, m=$ $1,2, \ldots, M_{1}$.

Logical Point Representation (Step 2). For segment $\mathcal{S}_{m}, m=1,2, \ldots, M$, we represent it as a logical point $p_{m}$. The traveling time by the WCV on segment $\mathcal{S}_{m}$ (excluding stopping time) is $D\left(\mathcal{S}_{m}\right) / V$. Denote $\omega\left(p_{m}\right)$ as the 
total stopping time that the $\mathrm{WCV}$ is in segment $\mathcal{S}_{m}$, i.e., $\omega\left(p_{m}\right)=\sum_{p \in \mathcal{S}_{m}}^{\omega(p)>0} \omega(p)$. Denote $\tau\left(p_{m}\right)$ as the total time that the WCV spends at $p_{m}$, which includes traveling time and stopping time. Then,

$$
\tau\left(p_{m}\right)=\frac{D\left(\mathcal{S}_{m}\right)}{V}+\sum_{p \in \mathcal{S}_{m}}^{\omega(p)>0} \omega(p)
$$

Note that energy consumption rate $C_{i B}(p)$ and energy charging rate $U_{i B}(p)$ may vary at different point in the same segment. To develop an $(1-\epsilon)$-optimal solution, we use the worst case values of energy consumption rate and energy charging rate within the segment to characterize a logical point. That is, for $i \in \mathcal{N}$, define $U_{i B}\left(p_{m}\right)$ and $C_{i B}\left(p_{m}\right)$ as

$$
U_{i B}\left(p_{m}\right) \triangleq \min _{p \in \mathcal{S}_{m}}\left\{U_{i B}(p)\right\}, C_{i B}\left(p_{m}\right) \triangleq \max _{p \in \mathcal{S}_{m}}\left\{C_{i B}(p)\right\} .
$$

Since there are $|\mathcal{N}|$ sensor nodes in the network, a logical point $p_{m}$ is represented by $2|\mathcal{N}|$-tuple vector $\left[U_{1 B}\left(p_{m}\right)\right.$, $\left.\ldots, U_{|\mathcal{N}| B}\left(p_{m}\right), C_{1 B}\left(p_{m}\right), \ldots, C_{|\mathcal{N}| B}\left(p_{m}\right)\right]$.

Find Lower and Upper Bounds (Step 3). Once we have a worst case representation of each logical point, we can obtain a formulation for a lower bound to OPT-s, which we denote as OPT-lb. Through a systematic change-of-variable technique, OPT-lb can be reformulated into an LP and can be solved in polynomial time (see [15] for more details).

Following the same token, we can find an upper bound to OPT-s by developing a best case representation for each logical point. This is done by defining

$$
\bar{U}_{i B}\left(p_{m}\right) \triangleq \max _{p \in p_{m}}\left\{U_{i B}(p)\right\}, \bar{C}_{i B}\left(p_{m}\right) \triangleq \min _{p \in \mathcal{S}_{m}}\left\{C_{i B}(p)\right\} .
$$

Based on this best-base representation, we obtain a formulation for an upper bound to OPT-s, denoted as OPT-ub. Similar to OPT-lb, OPT-ub can be reformulated into an LP and can be solved in polynomial time [15].

Termination Condition (Step 4) and Discretizing into Smaller Segments (Step 5). We can check the gap between the lower and upper bounds. If the gap is within desired accuracy (denoted by $\epsilon$ ), the algorithm terminates and returns an $(1-\epsilon)$-optimal solution; otherwise, we need to further discretize existing segments into smaller ones and perform another iteration and so forth.

For discretization, we denote $M_{i}, i=1,2, \cdots$, as the number of segments in the $i$-th iteration. By intuition, among all variables, $\omega\left(p_{m}\right)$ 's directly determine the WCV's stopping behavior at $p_{m}$ and thus the final objective value. Thus, we sort segments $p_{m}, m=1,2, \ldots, M_{i}$, in the decreasing value of $\omega\left(p_{m}\right)$ (a tie is broken randomly). From the ordered segments, we pick the first $|\mathcal{N}|$ segments and equally subdivide each of them into two segments. ${ }^{5}$ As a result, at most $|\mathcal{N}|$

\footnotetext{
${ }^{5}$ As there are $|\mathcal{N}|$ sensor nodes, the number of stops by the WCV (for charging) should be on the same order of $|\mathcal{N}|$. If $M_{i}<|\mathcal{N}|$, then we just divide all $M_{i}$ segments.
}

segments are added at each iteration, i.e., a linear increase in the number of segments.

Denote $\psi_{\mathrm{OPT}-\mathrm{lb}}^{\left(M_{i}\right)}$ and $\psi_{\mathrm{OPT}-\mathrm{ub}}^{\left(M_{i}\right)}$ as the optimal solutions to OPT-lb and OPT-ub at the end of the $i$-th iteration, respectively. Denote $\eta_{\text {vac }}^{\left(M_{i}\right)}$ and $\bar{\eta}_{\text {vac }}^{\left(M_{i}\right)}$ as the objective values in $\psi_{\text {OPT-lb }}^{\left(M_{i}\right)}$ and $\psi_{\mathrm{OPT}-\mathrm{ub}}^{\left(M_{i}\right)}$, respectively. Then we have the following lemma:

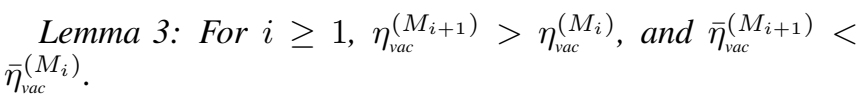

Lemma 3 says that as the number of segments increases with the number of iterations, the lower bound to OPT-s strictly increases while the upper bound strictly decreases. Lemma 3 can be proved by construction [15].

Recover a Feasible Solution to OPT-s (Step 6). Once the lower bound is within $(1-\epsilon)$ of the upper bound, the algorithm terminates. With the current lower bound solution to OPT-lb (denoted as $\psi_{\text {OPT-1b }}$ ), we can construct a feasible solution to OPT-s (denoted as $\psi_{\text {OPT-s }}$ ). Solution $\psi_{\text {OPT-s }}$ consists of several components, including (i) cycle time $\tau$; (ii) for $p \neq$ $p_{\text {vac }}$, stopping time $\omega(p)$, flow routing $f_{i j}(p)$ and $f_{i B}(p)$, and energy consumption rate $r_{i}(p)$; and (iii) vacation time at the service station $\tau_{\text {vac }}$, and corresponding flow routing $f_{i j}\left(p_{\text {vac }}\right)$ and $f_{i B}\left(p_{\text {vac }}\right)$, and energy consumption rate $r_{i}\left(p_{\text {vac }}\right)$. From $\psi_{\text {OPT-lb }}, \psi_{\text {OPT-s }}$ can be constructed as follows:

- It has the same cycle time $\tau$ as that in $\psi_{\text {OPT-lb }}$.

- For $p \neq p_{\text {vac }}$, the WCV may traverse segment $\mathcal{S}_{m}$ with or without any stop. To see if the WCV makes any stop in $\mathcal{S}_{m}$, we calculate $\tau\left(p_{m}\right)-D\left(\mathcal{S}_{m}\right) / V$. If the difference is greater than 0 , then the WCV stops within $\mathcal{S}_{m}$ and in $\psi_{\text {OPT-s }}$ the WCV may stop at any point $p \in \mathcal{S}_{m}$. For this $p$, the $\mathrm{WCV}$ will stay for $\tau\left(p_{m}\right)-D\left(\mathcal{S}_{m}\right) / V$ amount of time. Otherwise (i.e., $\left.\tau\left(p_{m}\right)-D\left(\mathcal{S}_{m}\right) / V=0\right)$, the WCV does not stop within $\mathcal{S}_{m}$. Regardless of stopping or not, the flow routing solution at a point $p \in \mathcal{S}_{m}$ is: $f_{i j}(p)=f_{i j}\left(p_{m}\right)$ and $f_{i B}(p)=f_{i B}\left(p_{m}\right)$. Corresponding to $p \in \mathcal{S}_{m}, r_{i}(p)$ is defined by (11).

- For $p=p_{\text {vac }}$, we have that $\tau_{\text {vac }}, f_{i j}\left(p_{\text {vac }}\right), f_{i B}\left(p_{\text {vac }}\right)$, and $r_{i}\left(p_{\text {vac }}\right)$ are the same as those in $\psi_{\text {OPT-lb }}$.

Denote $\eta_{\text {OPT-lb }}$ and $\eta_{\text {OPT-s }}$ as the objective values achieved by $\psi_{\text {OPT-lb }}$ and $\psi_{\text {OPT-s }}$, respectively. Since $\tau$ and $\tau_{\text {vac }}$ are unchanged in the foregoing solution construction, we have

$$
\eta_{\mathrm{OPT}-\mathrm{s}}=\eta_{\mathrm{OPT}-\mathrm{lb}}
$$

\section{Proof of $(1-\epsilon)$ Optimality}

Let $\eta_{\text {OPT-s }}^{*}$ be the (unknown) optimal objective value to OPT-s. The following theorem says that the constructed solution $\psi_{\text {OPT-s }}$ is $(1-\epsilon)$-optimal.

Theorem 2: For any $0<\epsilon \ll 1, \eta_{O P T-s} \geq(1-\epsilon) \eta_{O P T-s}^{*}$. 


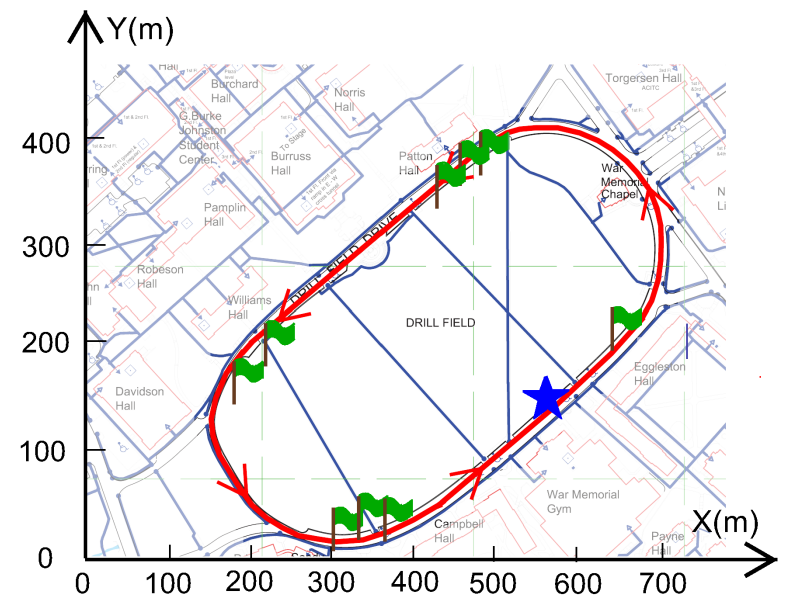

Fig. 4. Drillfield driveway on Virginia Tech campus. A star represents the WCV's home service station. A flag represents a stopping point in the case study (Section VII-B).

Proof: Upon termination, we have $\eta_{\mathrm{OPT}-\mathrm{lb}} \geq(1-\epsilon) \eta_{\mathrm{OPT}-\mathrm{ub}}$, where $\eta_{\mathrm{OPT}-\mathrm{lb}}$ and $\eta_{\mathrm{OPT} \text {-ub }}$ are the objective values achieved by the lower bound solution to OPT-lb and the upper bound solution to OPT-ub, respectively. Therefore, we have

$$
\eta_{\mathrm{OPT}-\mathrm{s}}=\eta_{\mathrm{OPT}-\mathrm{lb}} \geq(1-\epsilon) \eta_{\mathrm{OPT}-\mathrm{ub}} \geq(1-\epsilon) \eta_{\mathrm{OPT}-\mathrm{s}}^{*},
$$

where the first equality holds by (15), and the last inequality holds since $\eta_{\mathrm{OPT}-\mathrm{ub}}$ is an upper bound for OPT-s. This completes the proof.

\section{NUMERICAL RESULTS}

In this section, we use numerical results to demonstrate how our algorithm solves the WCV and mobile base station co-location problem.

\section{A. Network and Parameter Settings}

We use Virginia Tech's Drillfield (see Fig. 4) for sensor network deployment. Sensor nodes are deployed within a distance of the charging range along the side of the Drillfield driveway, which is roughly an ellipse. The home service station (marked as a star in Fig. 4) is located at $(540,160)$ (in m) along the driveway. For the Drillfield path $\mathcal{P}, D_{\mathcal{P}}=1228$ $\mathrm{m}$. The travel speed of the WCV is $V=5 \mathrm{~m} / \mathrm{s}$.

The number of sensor nodes in the network will be specified later for different results. The data rate $G_{i}, i \in \mathcal{N}$ for each node is randomly generated within $[1,10] \mathrm{Kb} / \mathrm{s}$. Suppose a sensor node uses a rechargeable NiMH battery (connected to an energy receiving coil). Assume $E_{\max }=10.8 \mathrm{~kJ}$ [7], and $E_{\min }=0.05 \cdot E_{\max }=540 \mathrm{~J}$. The power consumption coefficients are $\beta_{1}=50 \mathrm{~nJ} / \mathrm{b}, \beta_{2}=0.0013 \mathrm{pJ} /\left(\mathrm{b} \cdot \mathrm{m}^{4}\right)$, and $\rho=50 \mathrm{~nJ} / \mathrm{b}$ [13]. The path loss index is $\alpha=4$. For the charging efficiency function $\mu\left(D_{i B}\right)$, we refer to the experimental results in [6]. Through curve fitting to Fig. 3
TABLE I

LOCATION AND DATA RATE $G_{i}$ FOR EACH NODE IN A 25 -NODE NETWORK.

\begin{tabular}{|c|c|c||c|c|c|}
\hline $\begin{array}{c}\text { Node } \\
\text { Index }\end{array}$ & $\begin{array}{c}\text { Location } \\
(\mathrm{m})\end{array}$ & $\begin{array}{c}G_{i} \\
(\mathrm{~Kb} / \mathrm{s})\end{array}$ & $\begin{array}{c}\text { Node } \\
\text { Index }\end{array}$ & $\begin{array}{c}\text { Location } \\
(\mathrm{m})\end{array}$ & $\begin{array}{c}G_{i} \\
(\mathrm{~Kb} / \mathrm{s})\end{array}$ \\
\hline 1 & $(626.0,236.1)$ & 1 & 14 & $(247.6,181.6)$ & 7 \\
2 & $(623.3,235.6)$ & 7 & 15 & $(245.9,180.4)$ & 4 \\
3 & $(624.0,237.2)$ & 1 & 16 & $(247.7,181.0)$ & 10 \\
4 & $(625.6,237.1)$ & 1 & 17 & $(220.5,118.0)$ & 5 \\
5 & $(460.8,357.8)$ & 1 & 18 & $(220.5,121.1)$ & 2 \\
6 & $(462.6,361.9)$ & 4 & 19 & $(219.5,119.8)$ & 7 \\
7 & $(459.8,359.0)$ & 2 & 20 & $(220.7,118.3)$ & 6 \\
8 & $(461.1,359.0)$ & 3 & 21 & $(328.8,12.2)$ & 2 \\
9 & $(435.7,337.8)$ & 2 & 22 & $(335.2,13.2)$ & 9 \\
10 & $(433.3,337.7)$ & 4 & 23 & $(334.2,13.0)$ & 4 \\
11 & $(435.2,338.5)$ & 5 & 24 & $(333.2,13.9)$ & 8 \\
12 & $(434.8,337.4)$ & 5 & 25 & $(331.5,13.7)$ & 8 \\
13 & $(245.1,180.3)$ & 1 & & & \\
\hline
\end{tabular}

TABLE II

INDEX OF STOPPING POINT ALONG THE PATH, LOCATION AND TIME SPENT AT EACH STOPPING POINT FOR THE 25-NODE NETWORK.

\begin{tabular}{|c|c|c||c|c|c|}
\hline $\begin{array}{c}\text { Visit } \\
\text { Order }\end{array}$ & $\begin{array}{c}\text { Location } \\
(\mathrm{m})\end{array}$ & $\begin{array}{c}\omega(p) \\
(\mathrm{s})\end{array}$ & $\begin{array}{c}\text { Visit } \\
\text { Order }\end{array}$ & $\begin{array}{c}\text { Location } \\
(\mathrm{m})\end{array}$ & $\begin{array}{c}\omega(p) \\
(\mathrm{s})\end{array}$ \\
\hline 1 & $(625.7,235.3)$ & 23 & 6 & $(221.0,119.4)$ & 219 \\
2 & $(461.1,357.4)$ & 358 & 7 & $(329.3,11.9)$ & 2 \\
3 & $(464.5,360.2)$ & 9 & 8 & $(332.4,12.1)$ & 9 \\
4 & $(435.4,336.2)$ & 98 & 9 & $(333.9,12.4)$ & 2318 \\
5 & $(247.3,179.3)$ & 42 & & & \\
\hline
\end{tabular}

in [6], we obtain $\mu\left(D_{i B}\right)=-0.0958 D_{i B}^{2}-0.0377 D_{i B}+1.0$. Letting $U_{\max }=5 \mathrm{~W}$ and $\delta=1 \mathrm{~W}$, we have $D_{\delta}=2.7 \mathrm{~m}$ for a maximum distance of effective charging. ${ }^{6}$ We set $\epsilon=0.05$.

\section{B. A Case Study}

We first present results for a 25-node sensor network. The location of each node and its data rate are given in Table I. Applying our $(1-\epsilon)$-optimal algorithm, we find that the algorithm finds a solution after the third iteration. In this solution, we have $\tau=17.29 \mathrm{~h}, \tau_{\text {vac }}=16.29 \mathrm{~h}$, and the objective value is $94.21 \%{ }^{7}$ Since the total traveling time along path $\mathcal{P}$ is $1228 / 5=245.6 \mathrm{~s} \approx 0.07 \mathrm{~h}$, we have that the total stopping time for charging is $17.29-16.29-0.07=0.93 \mathrm{~h}$.

Upon termination, there are a total of 316 segments (corresponding to 316 logical points). However, the WCV only makes 9 stops among these segments, and merely traverses all the other segments without stop. For illustration purpose, we use a physical point $(x, y)$ within the corresponding segment to represent the segment where the WCV makes a stop. These stopping points are marked with flags in Fig. 4, and the location and the amount of time at each stop are given in Table II. Note that the number of stops for the WCV is much fewer than the number of sensor nodes due to multi-node charging. For example, the WCV charges nodes $1,2,3$, and 4

\footnotetext{
${ }^{6}$ The charging distance can be extended by using larger coils.

${ }^{7}$ The objective value achieved by the upper bound solution is $95.91 \%$. This means that our solution achieves an objective value that is at least $0.9421 / 0.9591=99.0 \%$ of the optimum.
} 
at the same time when it stops at the 1st point $(625.7,235.3)$. Also, it is possible that a node may be charged more than once in a cycle. For example, node 25 is charged when the WCV stops at both the 8th point $(332.4,12.1)$ and the 9th point $(333.9,12.4)$.

\section{CONCLUSIONS}

This paper explored the complex problem of co-locating a mobile base station on the wireless charging vehicle (WCV). The motivation was to take advantage of the mobility feature of the WCV and use it as a carrying platform for the mobile base station at the same time. The goal was to minimize energy consumption of the entire system while ensuring none of the sensor nodes runs out of energy. We developed a mathematical model for this problem and found that it is a highly complex problem involving time-dependent variables. Instead of studying the original problem formulation (OPT-t), we showed that it is sufficient to study a special subproblem (OPT-s) which only involves space-dependent variables. Subsequently, we developed a provable near-optimal solution to OPT-s. There are a number of novel techniques that are worth being disseminated to the research community, most notably, downsizing solution space without compromising optimal objective value, discretizing a continuous path into a finite number of segments and representing each segment with a logical point. These powerful techniques allow development of a provably near-optimal solution to a complex problem that is seemingly intractable at first glance. In our future work, we will further explore these techniques to address other difficult networking problems involving time-dependent movement, flow routing, and energy consumption.

\section{ACKNOWLEDGMENTS}

The work of Y.T. Hou and W. Lou was supported in part by NSF grants 0925719 (Hou), 1156311 (Lou) and 1156318 (Lou).

\section{REFERENCES}

[1] D. Ahn and S. Hong, "Effect of coupling between multiple transmitters or multiple receivers on wireless power transfer," to appear in IEEE Trans. on Industrial Electronics, available at http://ieeexplore.ieee.org/stamp/stamp.jsp?arnumber=06192319.

[2] J. Chang and L. Tassiulas, "Maximum lifetime routing in wireless sensor networks," IEEE/ACM Trans. on Networking, vol. 12, no. 4, pp. 609-619, Aug. 2004.
[3] S. He, J. Chen, F. Jiang, D.K.Y. Yau, G. Xing, and Y. Sun, "Energy provisioning in wireless rechargeable sensor networks," in Proc. IEEE INFOCOM, Shanghai, China, Apr. 2011, pp. 2006-2014.

[4] Y.T. Hou, Y. Shi, and H.D. Sherali, "Rate allocation and network lifetime problems for wireless sensor networks," IEEE/ACM Trans. on Networking, vol. 16, no. 2, pp. 321-334, Apr. 2008.

[5] A. Kurs, A. Karalis, R. Moffatt, J.D. Joannopoulos, P. Fisher, and M. Soljacic, "Wireless power transfer via strongly coupled magnetic resonances," Science, vol. 317, no. 5834, pp. 83-86, July 2007.

[6] A. Kurs, R. Moffatt, and M. Soljacic, "Simultaneous mid-range power transfer to multiple devices," Appl. Phys. Lett., vol. 96, pp. 044102-1044102-3, Jan. 2010.

[7] D. Linden and T.B. Reddy (eds.), Handbook of Batteries, 3rd ed., Chapter 1, McGraw-Hill, 2002.

[8] J. Luo and J.-P. Huabux, "Joint sink mobility and routing to maximize the lifetime of wireless sensor networks: The case of constrained mobility," IEEE/ACM Trans. on Networking, vol. 18, no. 3, pp. 871884, June 2010.

[9] R. C. Shah, S. Roy, S. Jain, and W. Brunette, "Data MULEs: Modeling a three-tier architecture for sparse sensor networks," in Proc. of First IEEE International Workshop on Sensor Network Protocols and Applications (SNPA), pp. 30-41, Anchorage, AK, May 2003.

[10] Y. Shi and Y.T. Hou, "Theoretical results on base station movement problem for sensor network," in Proc. IEEE INFOCOM, Phoenix, AZ, Apr. 2008, pp. 376-384.

[11] B. Tong, Z. Li, G. Wang, and W. Zhang, "How wireless power charging technology affects sensor network deployment and routing," in Proc. IEEE ICDCS, Genoa, Italy, Jun. 2010, pp. 438-447.

[12] U.S. Environmental Protection Agency and U.S. Department of Energy, "2013 Scion iQ EV," URL: http://www.fueleconomy.gov.

[13] L. Xie, Y. Shi, Y.T. Hou, and H.D. Sherali, "Making sensor networks immortal: An energy-renewal approach with wireless power transfer," IEEE/ACM Trans. on Networking, vol. 20, no. 6, pp. 1748-1761, Dec. 2012.

[14] L. Xie, Y. Shi, Y.T. Hou, W. Lou, H.D. Sherali, and S. F. Midkiff, "On renewable sensor networks with wireless energy transfer: The Multi-Node Case," in Proc. IEEE SECON, Seoul, Korea, June 2012, pp. 10-18.

[15] L. Xie, Y. Shi, Y.T. Hou, W. Lou, H.D. Sherali, and S. F. Midkiff, "Bundling mobile base station and wireless energy transfer: Modeling and optimization," Technical Report, the Bradley Department of Electrical and Computer Engineering, Virginia Tech, Blacksburg, VA, July 2012, available at http://filebox.vt.edu/users/windgoon/papers/TR12_Bundling.pdf.

[16] L. Xie, Y. Shi, Y.T. Hou, and W. Lou, "Wireless power transfer and applications to sensor networks," to appear in IEEE Wireless Communications Magazine, available at http://filebox.vt.edu/users/windgoon/papers/WCM13.pdf.

[17] G. Xing, T. Wang, W. Jia, and M. Li, "Rendezvous design algorithms for wireless sensor networks with a mobile base station," in Proc. ACM MobiHoc, Hong Kong, China, May 27-30, 2008, pp. 231-240.

[18] G.J. Zalmai, "Optimality conditions and Lagrangian duality in continuous-time nonlinear programming," Journal of Mathematical Analysis and Applications, vol. 109, no. 2, Aug. 1985, pp. 426-452.

[19] W. Zhao, M. Ammar, and E. Zegura, "A message ferrying approach for data delivery in sparse mobile ad hoc networks," in Proc. ACM MobiHoc, pp. 187-198, Tokyo, Japan, May 2004. 